\title{
Digital Transformation in the Area of Diabetes Management through Business Intelligence Technology
}

\author{
Tahani Daghistani \\ Information Systems and \\ Informatics Division (ISID) \\ Ministry of National \\ Guard-Health Affairs \\ Riyadh, Saudi Arabia
}

\author{
Huda Al Ghamdi \\ Information Systems and \\ Informatics Division (ISID) \\ Ministry of National \\ Guard-Health Affairs \\ Riyadh, Saudi Arabia
}

\author{
Abdullah Al Ghamdi \\ Family Medicine \\ Ministry of National \\ Guard-Health Affairs, \\ Riyadh \\ Saudi Arabia
}

\author{
Raed H. AlHazme \\ Information Systems and \\ Informatics Division (ISID) \\ Ministry of National \\ Guard-Health Affairs \\ Riyadh, Saudi Arabia
}

\begin{abstract}
Digital transformation and business intelligence in healthcare services for solving analytical tasks is a must. Analytic solutions continue to grow and will play an ever-increasing role in the healthcare future. Accordingly, healthcare organizations in quest of effective IT artifacts that will support the provision of a high quality care for patient based on accurate data analytics-based insights. In the area of diabetes management, there is a need for tools to track key indicators to support decision-making and provide elevated care. They require business intelligence for dashboards to visualize digitized data in order to gain insights regarding diabetes patient care and management. Simple and effective dashboards is mostly technology oriented to help physicians leverage patients' data most effectively.
\end{abstract}

\section{Keywords}

Digital Transformation, Business Intelligence, Dashboard, Data Analytics, Diabetes Management.

\section{INTRODUCTION}

The digital transformation enabled by business intelligence (BI) in healthcare field is transformative trend and seems to raise unabated. Digital transformation of healthcare has taken longer than other fields, large-scale adoption is necessary and radically will transform healthcare outcome for the better. These include weaving data and incorporate the use of business intelligence analytics to accelerate that transformation [1]. Advances in technology such as electronic medical records (EMRs) have enabled healthcare organizations to collect, store and manage effectively. However, they function more as data warehouses or repository than as dynamic analytic tools. The gap between collecting and analyzing data has to be addressed in order to bridging gap exist also between the received care and the actual care people received. The world of business intelligence solutions offering unparalleled insightful and actionable intelligence into organizations management and patients. Its effective means of healthcare transformation, the dynamic availability of analytics tools that hold great promise for improving health care. Analytics solution is increasingly merging itself into the arena of disease diagnosis and management in order to shape the future of healthcare. One major area where using analytics can optimize efforts is diabetes management hospital and foundation donations and grants $[2,3]$. From an IT point of view, business intelligence technology stays close to the data is dashboard, which is broad and far-reaching tool. Such enterprise data analytic tools used in support the transformation of data into information and analyzed to knowledge by visualizing and tracking metrics by healthcare organizations to support decision-making [4]. Viewing data this way, efforts on potential insights that can help physicians capture the patients with uncontrolled diabetes through easy tool then provide effective care to diabetes patients [3].

Overall, digital transformation and BI offer above all opportunities by digitized data availability, analytics, communicating information, along with emerging research trends such as personalized medicine. As a result, moves managing diabetes toward a new paradigm, success in providing a tailored care while revises practices, decisions, and treatments to individual in diabetic population. [5]. The impetus for this study is to explore the use of technology, in the form of business intelligence dashboard, can be adapted by a large, complex and multispecialty health care organization to potentially improve the overall care in particular diabetes management.

\section{RELATED WORK}

As mentioned in the introduction, the dashboard as business intelligence solution enables care professionals to identify uncontrolled diabetic patients and improve their care in an effective and time-efficient way. Most of the literature representing the value of dashboards without guarantee practice change; dashboards were not the only intervention for the use of the presented data.

Other strategies to improve quality of healthcare at population level are beyond the scope of this study, considerable efforts for diabetes management using dashboard summarized in this section.

Koopman et al. [6] compared the use of a new diabetes dashboard with the traditional electronic health record (EHR) search, which require viewing multiple screens or charts to retrieve data needed for diabetes care. The results quantified benefits of using patient-specific diabetes dashboard as decision support tool. The diabetes dashboard helps to ensure the availability of accurate data in a timely manner. Optimizing such health information technologies led to substantial improvements in the quality and cost of care. Dagliati et al. [7] implemented a dashboard-based system based on the clinical data from multiple-source and predictive analytics to support decision-making of T2DM and its complications. The results showed a decrease in visit duration, allow physicians to focus on the critical aspects of each patient. Thus, increase the efficiency of the clinical care process. Ngui and Qiu [8] developed an Electronic Medical Record (EMR) dashboard as health management approach for diabetes patients. They assessed the success of providing cardio protective to proper patients by using a dashboard. The 
results hold promise to improve treatment and engage patients while improving team-based care. Wu et al. [9] explored an approach to use database as a visual $\mathrm{HbA} 1 \mathrm{c}$ dashboard to effectively and timely flag patients with poor control and those who have missed their quarterly measurements. In the second phase, ongoing work to analyze the factors associated with uncontrolled diabetes. Such patient-centered tool enable patient to see their $\mathrm{HbA1c}$ trend to facilitate engagement and empower patients and their parents. Make data relevant for the clinic, patients and parents available, improving clinical outcomes. Giordanengo et, al. [10] designed dashboard to display data that collected from patients through systems like mobile apps to their clinicians. Moreover, they assessed the reliability of self-collected data including calculations, medical situations, and defined more data that meet clinicians' need. Such solution allow patients to be proactive toward disease management and allow clinicians to tailor health services to improve the patients' health state.

\section{METHODOLOGY}

A case study is the research methodology of this paper. Twocases designed dashboards explored; both contribute to diabetes management efforts, in King Abdulaziz Medical city (KAMC) setting. As a technical intervention, a developed analytics dashboard for diabetes patients that has been implemented in the department of family medicine and primary healthcare. The purpose of developed dashboard is to provide physicians, with a curated visual view of indicators related to the diabetes. Besides technical intervention, primary care provider assignment activated and improved in Electronic Medical Record (EMR) system including diabetes patients. Through these efforts, each patient will have a consistent continuity of care through a center point of care. Moreover, a developed analytics indicators that help to track and assess the results of the interventions. Then, a monitoring and evaluation dashboard have implemented to monitor the progress of PCP assignment. Additionally, we used several sources for data collection, well-documented reports and reviewing related articles. In addition, we conducted interviews, observations and attended meetings. The data collected between 2017 and 2018 .

\section{RESULTS}

The uncontrolled diabetes dashboard, produced by KAMC, is a web-based application accessed through an Internet browser (Figure 1). It was designed with the aim of offering insight into the diabetes patients' population in an integrated manner, together with flag uncontrolled diabetes where $\mathrm{HbAlc}$ is greater than or equal $8 \mathrm{mg} / \mathrm{dl}$, which would support physicians in their management of diabetes. This dashboard uses simple visualizations, and includes indicators that indicate particular test, which is HbA1c, has exceeded the recommended testing range. Then information of patients will be displayed included gender, age, routine clinical period, total patients and lab test for patients. Moreover, under this analytics page, the visualization features can be customized using different features such as date, facility type and location, region and patient type to populate the visualizations.

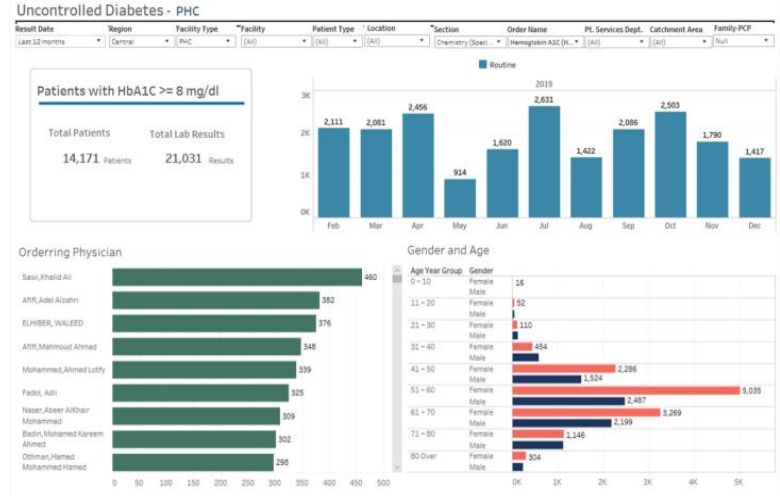

Fig 1: Uncontrolled diabetes dashboard that adopted in the family medicine/primary care at King Abdulaziz Medical City (KAMC)

The PCP dashboard is a good representation of the assignment progress of patients with a primary care physician. Key Metrics, direction and degree of progress across specific period of time. The dashboard tasked with determining number of registered patients over percentage of patients with primary care physicians. It designed to display a group of patients that can customized by date, region, and facility type (Figure 2).

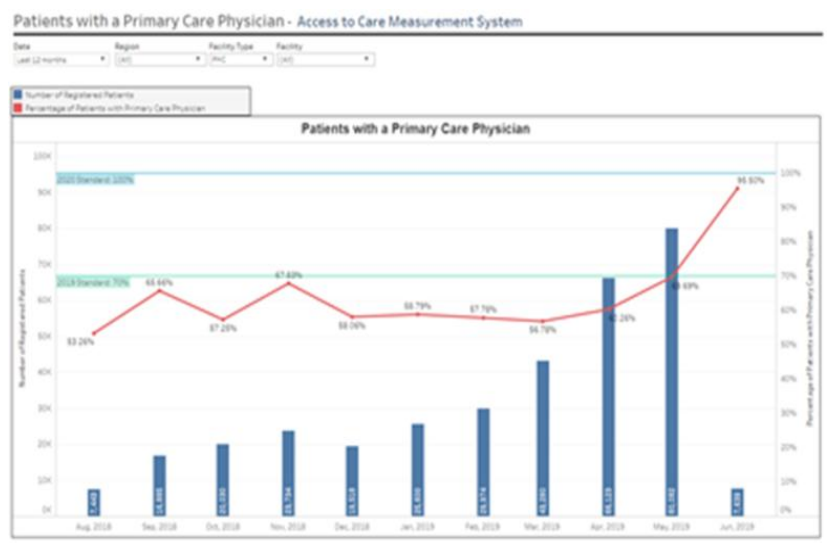

Fig 2: Patients with a primary care physician

Dashboard is an effective way to help control HbAlc and considered as an adjuvant technical intervention to the diabetes management. Given the reported clinical result, nominal cost, access to this technology, it expected to be effective at the population level. Comparisons before and after technical intervention in Figure 3. The result showed there is a significant improvement of the average of $\mathrm{HbAc1}$ in primary health care (PHC) after ten (10) months of enhancing the PCP assignment/developing the analytics dashboard to monitor PCP. Furthermore, t-test analysis (Figure 4) is showing a significant improvement in population health before 2017 and after 2018 intervention. 


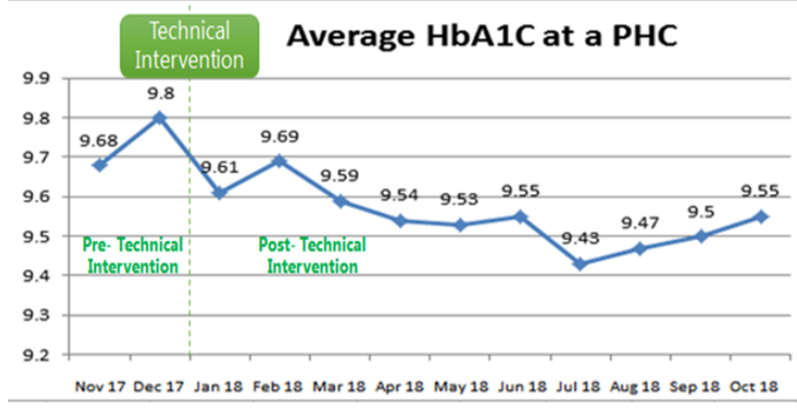

Fig 3: Average $\mathrm{HbA1C}$ at a PHC

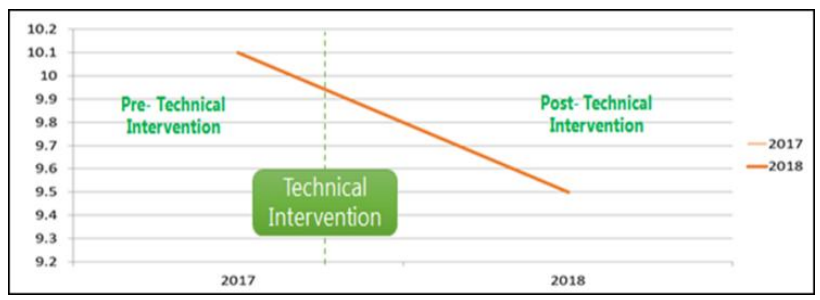

Fig 4: Population health - uncontrolled DM baseline before 2017 and after 2018 intervention

\section{DISCUSSION}

The first developed dashboard employs a unique strategy to motivate improvement of population health status. It aimed to flag uncontrolled diabetes where $\mathrm{HbAlc}$ is greater than or equal $8 \mathrm{mg} / \mathrm{dl}$ by displaying several measures of patients. This step is paired with encourage the assignment of all diabetes patients to a care provider. According to several studies, patients who see many primary care providers lead to inferior outcomes due to discontinuity of care. Alternatively, assigned patient to a care provider, who will know the patient well, improve communication and interaction, patients have the opportunity to articulate their problems and preferences to be considered in their treatment plans. Physicians provide patients with clear explanations and expectations to cope with their illness. Consequently, care providers will improve longterm ability to preserve an effective disease management [11]. The second dashboard, in this case study, that monitor assignment progress helps us understand the spots in terms of usage and interaction, which has an impact on assignments progression.

Technical intervention, such as dashboard, gave the opportunity of looking at health care from new perspectives, emphasizing temporal patterns of diabetes health status and facilitate action in response to the data have displayed. Adoption has been successful in in King Abdulaziz Medical city (KAMC) and they integrated its use into the routine clinic workflow. Usage and assignment progress has increased dramatically since the initial go-live a more than year ago. The potential of this technology to build the organization capabilities needed and a key driver to achieve the goals of Vision 2030", the National Transformation Program (NTP) 2020. The remarkable implementation of advanced information technology is a step to implement the plan of Saudi health-care digital transformational [12]. Correspondingly, it is important to monitor, track and assess the transformation and its progress for successful execution of the plan. The ability to judge the transformation to digital based process needs to present measurable outcomes. Therefore, taking into account using a rigorous mechanism to assess the progress of transformation by comparing before and after transformation within the organization [13]. Such efficient level by technology for digitization will give health care new appearance.

\section{CONCLUSION}

In conclusion, KAMC have implemented and shown that the use of the dashboard, which incorporates several features, can improve diabetes management. It anticipated that this dashboard would be helpful when adopted in a family medicine/ primary setting, where physicians can make quick decisions and provide additional attention to patients with diabetes based on summarized information. These evolving business intelligence technologies are already mad a positive impact. Smart leaders in healthcare arena must begin focusing and exploring on such technologies if they are to effectively navigate the digital transformation.

\section{REFERENCES}

[1] El Morr, C. and Ali-Hassan, H., 2019. Healthcare, Data Analytics, and Business Intelligence. In Analytics in Healthcare (pp. 1-13). Springer, Cham.

[2] Bellazzi, R., Dagliati, A., Sacchi, L. and Segagni, D., 2015. Big data technologies: new opportunities for diabetes management. Journal of diabetes science and technology, 9(5), pp.1119-1125.

[3] Sim, L.L.W., Ban, K.H.K., Tan, T.W., Sethi, S.K. and Loh, T.P., 2017. Development of a clinical decision support system for diabetes care: A pilot study. PloS one, 12(2), p.e0173021.

[4] Presthus, W. and Bergum, I., 2015. Business Intelligence to the People. A Case Study of Dashboard Adoption in the Health Care sector. In Nor. Konf. Organ. bruk av IT (Vol. 23, No. 1).

[5] Contreras, I. and Vehi, J., 2018. Artificial intelligence for diabetes management and decision support: literature review. Journal of medical Internet research, 20(5), p.e10775.

[6] Koopman, R.J., Kochendorfer, K.M., Moore, J.L., Mehr, D.R., Wakefield, D.S., Yadamsuren, B., Coberly, J.S., Kruse, R.L., Wakefield, B.J. and Belden, J.L., 2011. A diabetes dashboard and physician efficiency and accuracy in accessing data needed for high-quality diabetes care. The Annals of Family Medicine, 9(5), pp.398-405.

[7] Dagliati, A., Sacchi, L., Tibollo, V., Cogni, G., Teliti, M., Martinez-Millana, A., Traver, V., Segagni, D., Posada, J., Ottaviano, M. and Fico, G., 2018. A dashboard-based system for supporting diabetes care. Journal of the American Medical Informatics Association, 25(5), pp.538-547.

[8] Ngui, D., Lee, A. and Qiu, M., 2017. Using a Diabetes Electronic Medical Record (EMR) Dashboard to Improve Diabetes Care. Canadian Journal of Diabetes, 41(5), p.S53.

[9] Wu, Q., Huang, C., Parau, B. and Alexander, S., 2019, November. Development of a live visual HbA1c dashboard to improve engagement and clinical outcomes-a type 1 diabetes QI project. In 47th Meeting of the British Society for Paediatric Endocrinology and Diabetes (Vol. 66). BioScientifica.

[10] Giordanengo, A., Årsand, E., Woldaregay, A.Z., Bradway, M., Grottland, A., Hartvigsen, G., Granja, C., Torsvik, T. and Hansen, A.H., 2019. Design and 
Prestudy Assessment of a Dashboard for Presenting SelfCollected Health Data of Patients With Diabetes to Clinicians: Iterative Approach and Qualitative Case Study. JMIR diabetes, 4(3), p.e14002.

[11] Morrison, F., Shubina, M., Goldberg, S.I. and Turchin, A., 2013. Performance of primary care physicians and other providers on key process measures in the treatment of diabetes. Diabetes care, 36(5), pp.1147-1152.

[12] Vision2030. National Transformation Program. Delivery
Plan 2018-2020. Last accessed on 2019 July 25]. Available

from:

https://vision2030.gov.sa/en/programs/NTP

[13] Alharbi, M.F., 2018. An analysis of the Saudi health-care system's readiness to change in the context of the Saudi National Health-care Plan.

[14] In Vision 2030. International journal of health sciences, 12(3), p.83. 